\title{
The index and axial anomaly of a lattice Dirac operator
}

\author{
Ting-Wai Chiu ${ }^{\mathrm{a}}$ * \\ a Department of Physics, National Taiwan University, Taipei, Taiwan 106, Republic of China.
}

A remarkable feature of a lattice Dirac operator is discussed. Unlike the Dirac operator for massless fermions in the continuum, this Ginsparg-Wilson lattice Dirac operator does not possess topological zero modes for any topologically-nontrivial background gauge fields, even though it is exponentially-local, doublers-free, and reproduces correct axial anomaly for topologically-trivial gauge configurations.

\section{INTRODUCTION}

In the continuum, the Dirac operator of massless fermions in a smooth background gauge field with non-zero topological charge $Q$ has zero eigenvalues and the corresponding eigenfunctions are chiral. The Atiyah-Singer index theorem [1] asserts that the difference of the number of lefthanded and right-handed zero modes is equal to the topological charge of the gauge field configuration :

$n_{-}-n_{+}=Q$.

However, if one attempts to use the lattice to regularize the theory nonperturbatively, then not every lattice Dirac operator might possess topological zero modes ${ }^{2}$ with index satisfying (1), even if it is exponentially-local, doublers-free, and reproduces correct axial anomaly for topologicallytrivial gauge backgrounds. As a consequence, a topologically-trivial lattice Dirac operator might not realize 't Hooft's solution to the $U(1)$ problem in QCD, nor other quantities pertaining to the nontrivial gauge sectors. Nevertheless, from a theoretical viewpoint, it is interesting to realize that one may have the option to turn off the topological zero modes of a lattice Dirac operator, without affecting its correct behaviors ( axial anomaly, fermion propagator, etc. ) in the topologically-trivial gauge sector. In this talk, I

\footnotetext{
*'This work was supported by the National Science Council, R.O.C. under the grant numbers NSC89-2112-M002079 and NSC90-2112-M002-021

${ }^{2}$ So far, it has been confirmed that overlap Dirac operator $[3,4]$ and its generalization [5] can possess topological zero modes with index satisfying (1), on a finite lattice.
}

discuss an example of such lattice Dirac operators [6] and show that it does not possess topological zero modes for any topologically-nontrivial gauge configurations satisfying a very mild condition, Eq. (21).

\section{A LATTICE DIRAC OPERATOR}

Consider the lattice Dirac operator [6]

$$
D=a^{-1} D_{c}\left(\mathbf{I}+r D_{c}\right)^{-1}, \quad r=\frac{1}{2 c},
$$

with

$$
\begin{aligned}
& D_{c}=\sum_{\mu} \gamma^{\mu} T^{\mu}, T^{\mu}=f t^{\mu} f, \\
& f=\left(\frac{2 c}{\sqrt{t^{2}+w^{2}}+w}\right)^{1 / 2}, t^{2}=-\sum_{\mu} t^{\mu} t^{\mu} .
\end{aligned}
$$

Here $\gamma^{\mu} t^{\mu}$ is the naive lattice fermion operator and $-w$ is the Wilson term with a negative mass $-c(0<c<2)$

$$
\begin{aligned}
t^{\mu}(x, y)= & \frac{1}{2}\left[U_{\mu}(x) \delta_{x+\hat{\mu}, y}-U_{\mu}^{\dagger}(y) \delta_{x-\hat{\mu}, y}\right] \\
U_{\mu}(x)= & \exp \left[i a g A_{\mu}\left(x+\frac{a}{2} \hat{\mu}\right)\right] \\
w(x, y)= & c-\frac{1}{2} \sum_{\mu}\left[2 \delta_{x, y}+\right. \\
& \left.-U_{\mu}(x) \delta_{x+\hat{\mu}, y}-U_{\mu}^{\dagger}(y) \delta_{x-\hat{\mu}, y}\right]
\end{aligned}
$$

where the Dirac, color and flavor indices have been suppressed. Note that the $D_{c}$ defined in Eq. (3) can be regarded as a symmetrized version of that constructed in Ref. [7], for vector gauge theories. 
In the free fermion limit, (2) gives

$$
D(p)=D_{0}(p)+i \sum_{\mu} \gamma_{\mu} D_{\mu}(p)
$$

where

$$
\begin{aligned}
D_{0}(p) & =\frac{c}{a}\left(1-\frac{w(p)}{\sqrt{t^{2}(p)+w^{2}(p)}}\right) \\
D_{\mu}(p) & =\frac{c}{a} \frac{\sin \left(p_{\mu} a\right)}{\sqrt{t^{2}(p)+w^{2}(p)}}, \\
t^{2}(p) & =\sum_{\mu} \sin ^{2}\left(p_{\mu} a\right) \\
w(p) & =c-\sum_{\mu}\left[1-\cos \left(p_{\mu} a\right)\right] .
\end{aligned}
$$

Evidently, both $D_{0}(p)$ and $D_{\mu}(p)$ are analytic functions for all $p$ in the Brillouin zone. Thus

$$
D(x)=\int \frac{d^{4} p}{(2 \pi)^{4}} e^{i p \cdot x} D(p)
$$

is exponentially-local in the position space. The exponential locality of $D$ in the free fermion. limit immediately suggests that $D$ is also exponentially-local for sufficiently smooth background gauge fields.

It is easy to check that $D(p)$ is doublers-free and has the correct continuum behavior, i.e., in the limit $a \rightarrow 0$,

$D(p) \sim i \sum_{\mu} \gamma_{\mu} p_{\mu}+O\left(a p^{2}\right)$.

Further, $D$ is $\gamma_{5}$-hermitian,

$D^{\dagger}=\gamma_{5} D \gamma_{5}$,

and it breaks the chiral symmetry according to the Ginsparg-Wilson relation [2]

$D \gamma_{5}+\gamma_{5} D=2 \operatorname{raD} \gamma_{5} D$.

Thus $D$ satisfies the necessary requirements for a decent lattice Dirac operator.

The GW relation (16) immediately implies each zero mode of $D$ has a definite chirality, and the index of $D$ is equal to the sum of the axial anomaly $\operatorname{tr}\left[a \gamma_{5} D(x, x)\right]$ over all sites [8],

$$
\operatorname{index}(D)=n_{-}-n_{+}=r \sum_{x} \operatorname{tr}\left[\gamma_{5} a D(x, x)\right] \text {, }
$$

where the trace "tr" runs over the Dirac, color and flavor space.

However, the index relation (17) does not necessarily imply that $D$ can possess topological zero modes with the index satisfying (1). In fact, the GW Dirac operator (2) always has

$n_{+}=n_{-}=\sum_{x} \operatorname{tr}\left[a \gamma_{5} D(x, x)\right]=0$,

for any topologically-nontrivial gauge background, even though $D$ is exponentially-local, doublers-free, $\gamma_{5}$-hermitian, and has correct continuum behavior. The proof is as follows.

\section{A PROOF OF THE ABSENCE OF TOPOLOGICAL ZERO MODES}

From (15) and (16), we have

$$
D^{\dagger}+D=2 r a D^{\dagger} D=2 r a D D^{\dagger} \text {. }
$$

Thus $D$ is normal and $\gamma_{5}$-hermitian. Then the eigenvalues of $D$ are either real or in complex conjugate pairs. Each real eigenmode has a definite chirality, but each complex eigenmode has zero chirality. Further, the sum of the chirality of all real eigenmodes is zero ( chirality sum rule ) [9]. Now the eigenvalues of $D(2)$ fall on a circle in the complex plane, with center $(c / a, 0)$ on the real axis, and radius of length $c / a$. Then the chirality sum rule reads

$n_{+}-n_{-}+N_{+}-N_{-}=0$,

where $n_{+}\left(n_{-}\right)$denotes the number of zero modes of positive (negative ) chirality, and $N_{+}\left(N_{-}\right)$the number of nonzero ( eigenvalue $2 c / a$ ) real eigenmodes of positive (negative) chirality.

The chirality sum rule (20) asserts that each topological zero mode must be accompanied by a nonzero real eigenmode with opposite chirality, and vice versa. ( Note that both topological zero modes and their corresponding nonzero real eigenmodes are robust under local fluctuations of the gauge background, thus one can easily distinguish them from those trivial zero and nonzero real eigenmodes which are unstable under local fluctuations of the background ).

It follows that if $D$ cannot have any nonzero real eigenmodes in topologically nontrivial gauge 
backgrounds, then $D$ cannot possess any topological zero modes.

From (2), any zero mode of $D$ is also a zero mode of $D_{c}$, and vice versa. However, a nonzero real ( eigenvalue $2 c / a$ ) eigenmode of $D$ corresponds to a pole ( singularity) in the spectrum of $D_{c}$, since $D_{c}=D(\mathbf{I}-r a D)^{-1}$, the inverse tranform of (2).

Therefore, if the spectrum of $D_{c}$ does not contain any poles ( singularities ) for a topologicallynontrivial gauge background, then $D$ cannot have any nonzero real eigenmodes, thus no topological zero modes.

Now we consider topologically-nontrivial gauge configurations satisfying the condition ${ }^{3}$

$\operatorname{det}\left(\sqrt{t^{2}+w^{2}}+w\right) \neq 0$.

Then $f$ exists, and $D_{c}(3)$ is well-defined ( without any poles ). It follows that $D(2)$ cannot have topological zero modes for any topologicallynontrivial gauge configurations satisfying (21). This completes the proof.

\section{AXIAL ANOMALY}

From (18), the topological triviality of $D$ (2) implies that it cannot reproduce correct axial anomaly for topologically-nontrivial backgrounds. Nevertheless, since $D$ is exponentiallylocal, doublers-free and has correct continuum behavior, these conditions are sufficient to ensure that it reproduces continuum axial anomaly for topologically-trivial gauge backgrounds. The axial anomaly of (2) has been calculated [10] in weak coupling perturbation theory, up to $O\left(g^{4}\right)$ of the gauge coupling $g$, for toplogically-trivial gauge configurations. It has been shown that the axial anomaly recovers the topological charge density in the continuum limit, i.e.,

$$
\begin{aligned}
\mathcal{A}(x) & =\operatorname{tr}\left[\gamma_{5}(\mathbf{I}-r a D)(x, x)\right] \\
& =\frac{g^{2}}{32 \pi^{2}} \sum_{\mu \nu \lambda \sigma} \epsilon_{\mu \nu \lambda \sigma} \operatorname{tr}\left(F_{\mu \nu} F_{\lambda \sigma}\right)+O(a),
\end{aligned}
$$

where $F_{\mu \nu}=\partial_{\mu} A_{\nu}-\partial_{\nu} A_{\mu}+i g\left[A_{\mu}, A_{\nu}\right]$.

\footnotetext{
${ }^{3}$ It should be emphasized that we have not found any robust nontrivial gauge configuration violating (21), on a finite lattice. Thus, it is likely that the measure of the nontrivial gauge configurations not satisfying (21) is zero.
}

\section{CONCLUSIONS}

For some years, it has been taken for granted that if a Ginsparg-Wilson lattice Dirac operator has correct axial anomaly for the trivial gauge sector, then it must also reproduce continuum axial anomaly for the nontrivial sectors. However, the lattice Dirac operator (2) provides a counterexample, and suggests that this common conception may not be justified.

In general, given a topologically-proper lattice Dirac operator, it can be transformed into a topologically-trivial lattice Dirac operator which is identical to the topologically-proper one in the free fermion limit. On the other hand, given a topologically-trivial GW Dirac operator, it remains an interesting question how to transform it into a topologically-proper one.

If one insists that the topologically zero modes of a lattice Dirac operator are crucial for lattice QCD to reproduce the low energy hadron phenomenology, then one should assure that a Ginsparg-Wilson lattice Dirac operator is indeed topologically-proper, before it could be employed for lattice QCD computations. However, there might be a very slight possibility that lattice QCD with topologically-trivial quarks could reproduce low energy hadron phenomenology. These issues deserve further studies.

\section{REFERENCES}

1. M. F. Atiyah and I. M. Singer, Annals Math. 87 (1968) 484; ibid, 93 (1971) 119.

2. P. H. Ginsparg and K. G. Wilson, Phys. Rev. D 25 (1982) 2649.

3. H. Neuberger, Phys. Lett. B 417 (1998) 141.

4. R. Narayanan and H. Neuberger, Nucl. Phys. B 443 (1995) 305.

5. K. Fujikawa, Nucl. Phys. B 589 (2000) 487.

6. T.W. Chiu, Phys. Lett. B 521 (2001) 429.

7. T.W. Chiu, Phys. Lett. B 467 (1999) 112.

8. P. Hasenfratz, V. Laliena and F. Niedermayer, Phys. Lett. B 427 (1998) 125.

9. T.W. Chiu, Phys. Rev. D 58 (1998) 074511; Nucl. Phys. B 588 (2000) 400.

10. T.W. Chiu and T.H. Hsieh, hep-lat/0109016. 